\title{
A New Technique for Simulation the Zakharov-Kuznetsov Equation
}

\author{
A. S. J. Al-Saif ${ }^{1}$, Mohammed S. Abdul-Wahab² \\ Department of Mathematics, College of Education for Pure Sciences, University of Basrah, Basrah, Iraq ${ }^{1,2}$ \\ sattaralsaif@yahoo.com¹,moh111sbah@gmail.com²
}

\begin{abstract}
In this article, a new technique is proposed to simulated two-dimensional Zakharov-Kuznetsov equation with the initial condition. The idea of this technique is based on Taylors' series in its derivation. Two test problems are presented to illustrate the performance of the new scheme. Analytical approximate solutions that we obtain are compared with variational iteration method (VIM) and homotopy analysis method (HAM). The results show that the new scheme is efficient and better than the other methods in accuracy and convergence.
\end{abstract}

Keywords: Taylors' Series, Zakharov-Kuznetsov Equation, Simulation, Analytical Solution, Accuracy, Convergence.

Mathematics Subject Classifications 2010 [MSC]: 76S05, 65N99, 35Q35

Date of Publication: 2018-09-30

DOI: https://doi.org/10.24297/jam.v14i2.7559

ISSN: 2347-1921

Volume: 14 Issue: 02

Journal: Journal of Advances in Mathematics

Website: https://cirworld.com

This work is licensed under a Creative Commons Attribution 4.0 International License. 


\section{1- Introduction}

The nonlinear partial differential equations represent the modeling of many phenomena in various fields such as mathematics, physics, chemistry, engineering, biology, astronomy and fluids mechanics etc. The ZakharovKuznetsov (ZK) equation is one of them, it appears in plasma physics [1]. This equation has attracted the attention of many researchers in the last years. They are trying to solve it by using different methods. For example, the extended tanh method (Wazwaz in 2008 [2]), VIM (Molliq et al. in 2009 [3]), He's homotopy perturbation method (Yildirim et al. in 2010 [4]), Lie group analysis (Khalique et al. in 2011 [5]), the improved (G'/G)-expansion method (Naher et al. in 2012 [6]), traveling wave technique (Arshad et al. in 2016[7] and Yuan et al. in 2013 [8]), extended direct algebraic method (Seadawyin 2014 [9]), fractional sub-equation method (Saha et al. in 2015 [10]), homotopy perturbation method (Jamshad et al. in 2017 [11]), and solitary wave (Zhongzhou et al. in 2018 [12]). In this article, we study two-dimensional Zakharov-Kuznetsov equation:

$u_{t}+p_{1}\left(u^{q_{1}}\right)_{x}+p_{2}\left(u^{q_{2}}\right)_{x x x}+p_{3}\left(u^{q_{3}}\right)_{y y x}=0$,

where $u=u(x, y, t),\left\{p_{1}, p_{2}, p_{3}\right\}$ are arbitrary constants and $\left\{q_{1}, q_{2}, q_{3}\right\}$ are integers. This equation governs the behavior of weakly nonlinear ion-acoustic waves in plasma comprising cold ions and hot isothermal electrons in the presence of a uniform magnetic field $[13,14]$. The main aim of this work is to find analytical approximate solutions to equation (1.1) by using a new simulation scheme that is considered as extending and developing to that in [15]. This scheme is based on the Taylor series, it is efficient to solve linear and nonlinear equations. Also, our survey reveals that no attempt has ever been made to study the current model by using this technique. These reasons stimulate us to employ it to solve nonlinear intricate problem such as the twodimensional Zakharov-Kuznetsov equation. The obtained results are perfect, have small absolute errors and the best compared with VIM and HAM.

This paper is organized as follows: Generating a simulation scheme in section 2, two problems are tested in section 3, in section 4 the convergence analysis is presented, discussion of results is reported in section 5 . Finally, the conclusions are recorded in section 6 .

\section{2- Generating a simulation scheme}

In this section, the basic ideas for constructing a new simulation scheme will be discussed.

Let's consider the initial value problem:

$u_{t}(x, y, t)=F[u]+g(x, y)$

with initial condition $u\left(x, y, t_{0}\right)$,

where $F[u]$ is the linear and nonlinear operator and $g(x, y)$ is the known function.

By using the integral for the two sides of equation (2.1) from $t_{0}$ to $t$, we obtain

$u(x, y, t)=u\left(x, y, t_{0}\right)+g(x, y) \Delta t+\int_{t_{0}}^{t} F[u] d t$,

where $\Delta t=t-t_{0}$, and $F[u]$ can be expressed by the expand Taylors' series about $t_{0}$ as;

$$
\begin{aligned}
& F[u]=[F[u]]_{t_{0}}+\left[F^{\prime}[u]\right]_{t_{0}} \frac{\Delta t}{1 !}+\left[F^{\prime \prime}[u]\right]_{t_{0}} \frac{(\Delta t)^{2}}{2 !}+\left[F^{\prime \prime \prime}[u]\right]_{t_{0}} \frac{(\Delta t)^{3}}{3 !}+\cdots+\left[F^{(n)}[u]\right]_{t_{0}} \frac{(\Delta t)^{n}}{n !}+\cdots, \\
& \text { where }[F[u]]_{t_{0}}=\left.F[u]\right|_{t=t_{0^{\prime}}}\left[F^{\prime}[u]\right]_{t_{0}}=\left.\frac{\partial F[u]}{\partial t}\right|_{t=t_{0}},\left[F^{\prime \prime}[u]\right]_{t_{0}}=\left.\frac{\partial^{2} F[u]}{\partial t^{2}}\right|_{t=t_{0}}, \cdots,\left[F^{(n)}[u]\right]_{t_{0}}=\left.\frac{\partial^{n} F[u]}{\partial t^{n}}\right|_{t=t_{0}}
\end{aligned}
$$


Substituting equation (2.3) into equation (2.2), and integrating resulting equation to obtain the series solution as;

$$
\begin{aligned}
& u(x, y, t)=a_{0}+a_{1} \Delta t+a_{2} \frac{(\Delta t)^{2}}{2 !}+a_{3} \frac{(\Delta t)^{3}}{3 !}+\cdots+a_{n} \frac{(\Delta t)^{n}}{n !}+\cdots, \\
& \text { where } a_{0}=u\left(x, y, t_{0}\right), a_{1}=g(x, y)+[F[u]]_{t_{0}} a_{2}=\left[F^{\prime}[u]\right]_{t_{0}} a_{3}=\left[F^{\prime \prime}[u]\right]_{t_{0}}, \ldots, a_{n}=\left[F^{(n-1)}[u]\right]_{t_{0}} .
\end{aligned}
$$

Here we used the chain rule to compute the derivatives of $F[u]$

$$
\begin{aligned}
& F^{\prime}[u]=\sum_{i=0}^{n} \sum_{j=0}^{i} F_{u_{x^{i-j} j} j}[u] u_{x^{i-j} y^{j} t} \\
& F^{\prime \prime}[u]=\sum_{i=0}^{n} \sum_{j}^{i}\left(F_{u_{x^{i-j} y^{j}}}[u] u_{x^{i-j} y^{j} t t}+\sum_{k=0}^{n} \sum_{r=0}^{k} F_{\left(u_{x^{i-j} y^{j}}\right),\left(u_{x^{k-r} y^{r}}\right)}[u] u_{x^{i-j} y^{j}} u_{x^{k-r} y^{r} t}\right), \\
& \quad \vdots
\end{aligned}
$$

where $n$ is the highest derivative of $u$.

The series solution (2.4) at an initial time $\left(t_{0}=0\right)$ is

$$
u(x, y, t)=a_{0}+a_{1} t+a_{2} \frac{t^{2}}{2 !}+a_{3} \frac{t^{3}}{3 !}+\cdots+a_{n} \frac{t^{n}}{n !}+\cdots .
$$

\section{3- Test Problems}

Example 1. [16] Consider the following equation

$u_{t}+\left(u^{2}\right)_{x}+\frac{1}{8}\left(u^{2}\right)_{x x x}+\frac{1}{8}\left(u^{2}\right)_{y y x}=0$

with initial condition $u(x, y, 0)=\frac{4}{3} k \sinh ^{2}(x+y)$. The exact solution for this problem is $u(x, y, t)=\frac{4}{3} k \sinh ^{2}(x+y-k t)$, compare with equation (2.1), we have

$g(x, y)=0$,

$F[u]=-\left(u^{2}\right)_{x}-\frac{1}{8}\left(u^{2}\right)_{x x x}-\frac{1}{8}\left(u^{2}\right)_{y y x}$,

we note that the highest derivative of $u$ is $n=3$ and $t_{0}=0$, then according to (2.5), we get

$$
\begin{aligned}
a_{0} & =u(x, y, 0)=\frac{4}{3} k \sinh ^{2}(x+y), \\
a_{1} & =[F[u]]_{0}=-\left(\left(a_{0}\right)^{2}\right)_{x}-\frac{1}{8}\left(\left(a_{0}\right)^{2}\right)_{x x x}-\frac{1}{8}\left(\left(a_{0}\right)^{2}\right)_{y y x} \\
& =-\frac{32}{9} k^{2} \sinh (x+y) \cosh (x+y)\left[10 \cosh ^{2}(x+y)-7\right], \\
a_{2} & =\left[F^{\prime}[u]\right]_{0}=\sum_{i=0}^{3} \sum_{j=0}^{i} F_{u_{x^{i-j} y^{j}}}\left[a_{0}\right]\left(a_{1}\right)_{x^{i-j} y^{j}} \\
& =\frac{128}{27} k^{3}\left[1200 \cosh ^{6}(x+y)-2080 \cosh ^{4}(x+y)+968 \cosh ^{2}(x+y)-79\right], \\
a_{3} & =\left[F^{\prime \prime}[u]\right]_{0}=\sum_{i=0}^{3} \sum_{j}^{i}\left(F_{u_{x^{i-j} y^{j}}}\left[a_{0}\right]\left(a_{2}\right)_{x^{i-j} y^{j}}+\sum_{k=0}^{3} \sum_{r=0}^{k} F_{\left(u_{x^{i-j} y^{j}}\right),\left(u_{x^{k-r} y^{r}}\right)}\left[a_{0}\right]\left(a_{1}\right)_{x^{i-j} y^{j}}\left(a_{1}\right)_{x^{k-r} y^{r}}\right)
\end{aligned}
$$




$$
\begin{aligned}
= & -\frac{8192}{81} k^{4} \sinh (x+y) \cosh (x+y)\left[23800 \cosh ^{6}(x+y)-42900 \cosh ^{4}(x+y)\right. \\
& \left.+22665 \cosh ^{2}(x+y)-3142\right],
\end{aligned}
$$

from equation (2.8) we get the analytical approximate solution $\quad u(x, y, t)=\sum_{i=0}^{3} a_{i} \frac{(t)^{i}}{(i) !}$.

Example 2. [16] Consider the following equation

$u_{t}+\left(u^{3}\right)_{x}+2\left(u^{3}\right)_{x x x}+2\left(u^{3}\right)_{y y x}=0$,

with initial condition $u(x, y, 0)=\frac{3}{2} k \sinh \left(\frac{1}{6}(x+y)\right)$. The exact solution for this problem is $u(x, y, t)=\frac{3}{2} k \sinh \left(\frac{1}{6}(x+y-k t)\right)$, compare with equation (2.1), we have

$g(x, y)=0$

$F[u]=-\left(u^{3}\right)_{x}-2\left(u^{3}\right)_{x x x}-2\left(u^{3}\right)_{y y x}$,

we note that the highest derivative of $u$ is $n=3$ and $t_{0}=0$, then according to (2.5), we get

$$
\begin{aligned}
a_{0}= & u(x, y, 0)=\frac{3}{2} k \sinh \left(\frac{1}{6}(x+y)\right), \\
a_{1}= & {[F[u]]_{0}=-\left(\left(a_{0}\right)^{3}\right)_{x}-2\left(\left(a_{0}\right)^{3}\right)_{x x x}-2\left(\left(a_{0}\right)^{3}\right)_{y y x} } \\
= & -\frac{27}{8} k^{3} \cosh ^{3}\left(\frac{1}{6}(x+y)\right)+3 k^{3} \cosh \left(\frac{1}{6}(x+y)\right), \\
a_{2}= & {\left[F^{\prime}[u]\right]_{0}=\sum_{i=0}^{3} \sum_{j=0}^{i} F_{u_{x^{i-j} y^{j}}}\left[a_{0}\right]\left(a_{1}\right)_{x^{i-j} y^{j}} } \\
= & \frac{3}{32} k^{5} \sinh \left(\frac{1}{6}(x+y)\right)\left[765 \cosh ^{4}\left(\frac{1}{6}(x+y)\right)-729 \cosh ^{2}\left(\frac{1}{6}(x+y)\right)+91\right], \\
a_{3}= & {\left[F^{\prime \prime}[u]\right]_{0}=\sum_{i=0}^{3} \sum_{j}^{i}\left(F_{u_{x^{i-j}} j}\left[a_{0}\right]\left(a_{2}\right)_{x^{i-j} y^{j}}+\sum_{k=0}^{3} \sum_{r=0}^{k} F_{\left(u_{x^{i-j}} j\right.}\right),\left(u_{x^{k-r} y^{r}}\left[a_{0}\right]\left(a_{1}\right)_{x^{i-j} y^{j}}\left(a_{1}\right)_{x^{k-r} y^{r}}\right) } \\
= & -\frac{3}{128} k^{7} \cosh \left(\frac{1}{6}(x+y)\right)\left[188181 \cosh ^{6}\left(\frac{1}{6}(x+y)\right)-382293 \cosh ^{4}\left(\frac{1}{6}(x+y)\right)\right. \\
& \left.+234468 \cosh h^{2}\left(\frac{1}{6}(x+y)\right)-39851\right],
\end{aligned}
$$

from equation (2.8) we get the analytical approximate solution $u(x, y, t)=\sum_{i=0}^{3} a_{i} \frac{(t)^{i}}{(i) !}$. 
Table 1: Comparison of the absolute errors between VIM [17] and present study for example 1 with $t=1, k=$ 0.001 .

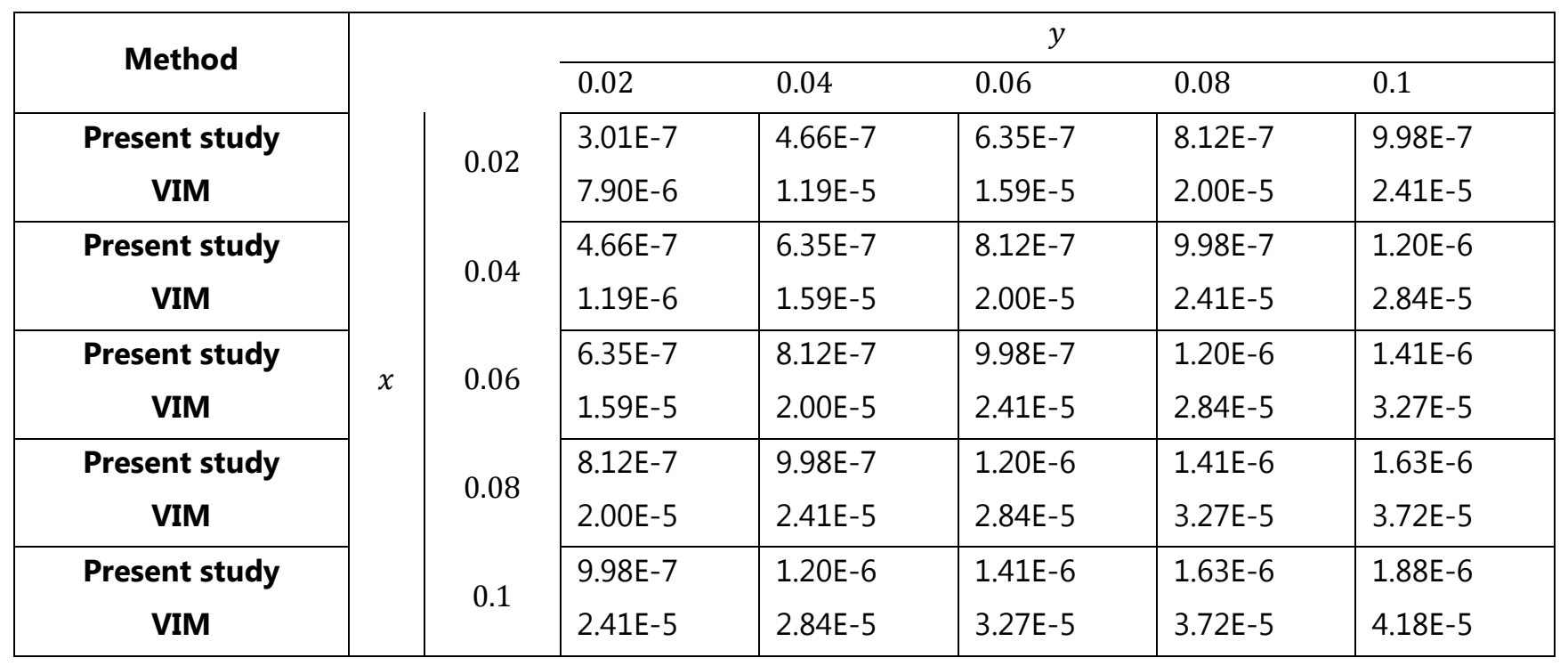

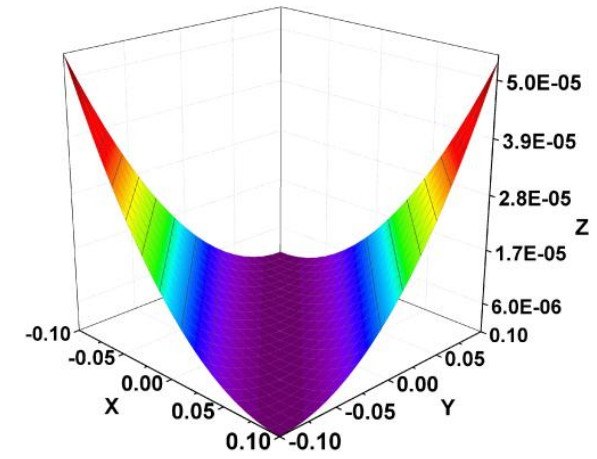

(a)

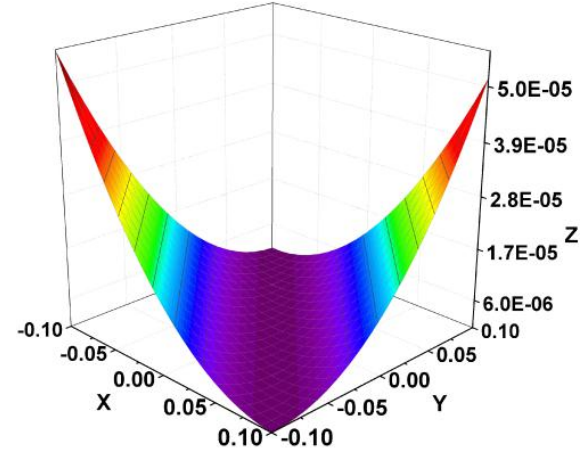

(b)

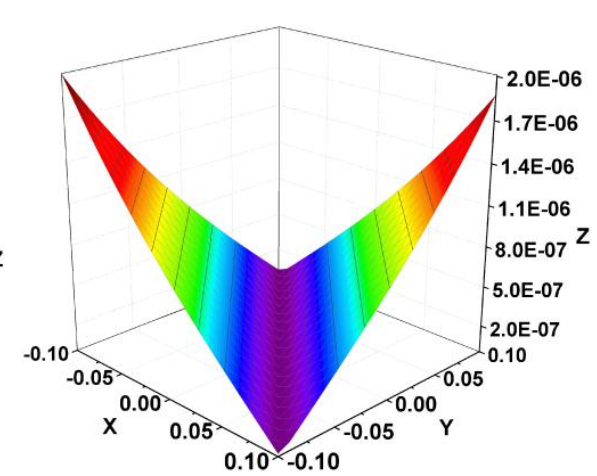

(c)

Fig. 1: (a) Exact solution, (b) Approximate solution, (c) Absolute errors for $t=1$ with $k=0.001$.

Table 2: Comparison of the absolute errors between HAM [18] and present study for example 2 with $t=1$, $k=0.001$.

\begin{tabular}{|c|c|c|c|c|c|c|c|}
\hline \multirow{2}{*}{ Method } & \multirow{12}{*}{$x$} & & \multicolumn{5}{|c|}{$y$} \\
\hline & & \multirow{3}{*}{1} & 1 & 3 & 5 & 7 & 9 \\
\hline Present study & & & $2.63 \mathrm{E}-7$ & $3.05 E-7$ & $3.78 \mathrm{E}-7$ & $4.85 E-7$ & $6.24 \mathrm{E}-7$ \\
\hline HAM & & & $2.64 \mathrm{E}-7$ & $3.08 \mathrm{E}-7$ & $3.86 \mathrm{E}-7$ & 5.07E-7 & $6.85 E-7$ \\
\hline Present study & & 2 & $3.05 \mathrm{E}-7$ & $3.78 \mathrm{E}-7$ & $4.85 \mathrm{E}-7$ & $6.24 \mathrm{E}-7$ & $7.72 \mathrm{E}-7$ \\
\hline HAM & & & $3.08 \mathrm{E}-7$ & $3.86 \mathrm{E}-7$ & $5.07 \mathrm{E}-7$ & $6.85 E-7$ & $9.40 \mathrm{E}-7$ \\
\hline Present study & & $\Gamma$ & $3.78 \mathrm{E}-7$ & $4.85 E-7$ & $6.24 \mathrm{E}-7$ & $7.72 \mathrm{E}-7$ & $8.41 E-7$ \\
\hline HAM & & & $3.86 \mathrm{E}-7$ & 5.07E-7 & $6.85 \mathrm{E}-7$ & $9.40 \mathrm{E}-7$ & $1.30 \mathrm{E}-6$ \\
\hline Present study & & 7 & $4.85 \mathrm{E}-7$ & $6.24 \mathrm{E}-7$ & $7.72 \mathrm{E}-7$ & $8.41 \mathrm{E}-7$ & $5.54 \mathrm{E}-7$ \\
\hline HAM & & & $5.07 E-7$ & $6.85 \mathrm{E}-7$ & $9.40 \mathrm{E}-7$ & $1.30 \mathrm{E}-6$ & $1.80 \mathrm{E}-6$ \\
\hline Present study & & 0 & $6.24 \mathrm{E}-7$ & $7.72 E-7$ & $8.41 \mathrm{E}-7$ & $5.54 \mathrm{E}-7$ & $8.95 E-7$ \\
\hline HAM & & & $6.85 \mathrm{E}-7$ & $9.40 \mathrm{E}-7$ & $1.30 \mathrm{E}-6$ & $1.80 \mathrm{E}-6$ & $2.50 \mathrm{E}-6$ \\
\hline
\end{tabular}




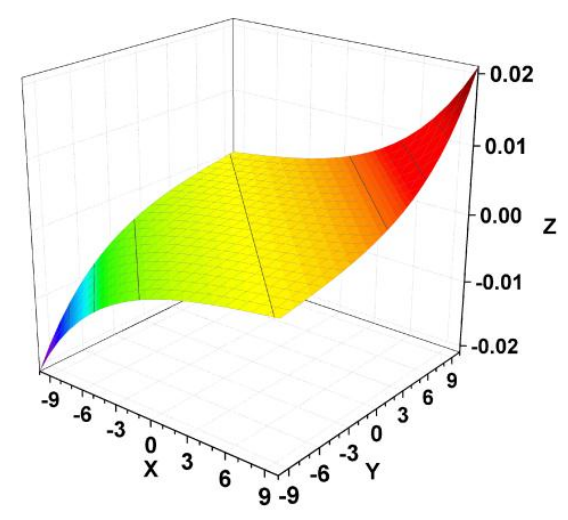

(a)

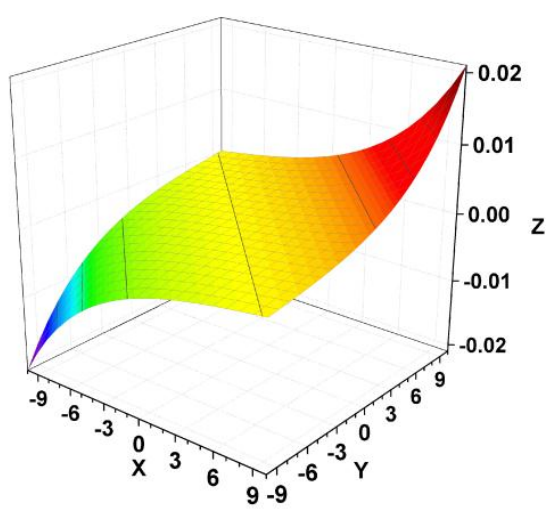

(b)

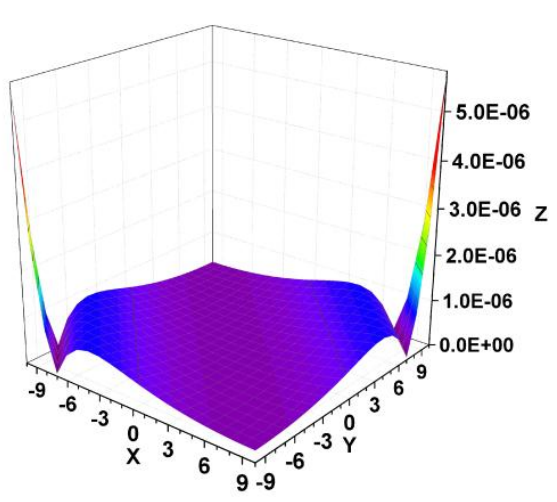

(c)

Fig. 2: (a) Exact solution, (b) Approximate solution, (c) Absolute errors for $t=1$ with $k=0.001$.

\section{4- Discussion}

In this article, we introduced two test problems for confirming the validity of the new proposed approach. Figs. (1-2) show the exact solution, approximate solution and absolute errors at $t=1, k=0.001$, and the comparison between analytical approximate solution obtain by a new approach and VIM [17] and HAM [18] which are given in Tables (1-2). The measurement of errors for the unknown variable, which are shown in Tables (1-2), ensure the ability of the suggested new approach and its accuracy in finding the analytical approximate solutions of nonlinear two-dimensional Zakharov-Kuznetsov equation. From our computations that are explained in the figures and tables, we noted that the analytical approximate solution obtained by a new approach is identical with exact solutions. Moreover, the absolute errors of the proposed approach are smaller than other standard methods (VIM and HAM). In addition, theoretical proofs for the analysis of convergence stand by the computation results. From these results, we can say that, the power series simulation scheme is an effective and good approach to find the solutions of nonlinear two-dimensional Zakharov-Kuznetsov equation compared to the other methods (VIM, HAM).

\section{5- Convergence analysis}

Consider the partial differential equation (1.1) in the following form:

$u(x, y, t)=G(u(x, y, t))$,

where $G$ is a nonlinear operator. The solution by the present approach is equivalent to the following sequence:

$S_{n}=\sum_{i=0}^{n} u_{i}=\sum_{i=0}^{n} a_{i} \frac{(\Delta t)^{i}}{(i) !}$.

\section{Theorem 4.1 ( Convergence of Zakharov-Kuznetsov equation )}

Let $G$ be an operator from a Hilbert space $H$ into $H$ and $u$ be the exact solution of equation (4.1). The approximate solution $\sum_{i=0}^{\infty} u_{i}=\sum_{i=0}^{\infty} a_{i} \frac{(\Delta t)^{i}}{(i) !}$ is Convergence to exact solution $u$

when $\exists 0 \leq \alpha<1,\left\|u_{i+1}\right\| \leq \alpha\left\|u_{i}\right\| \forall i \in \mathbb{N} \cup\{0\}$.

Proof: We want to show that $\left\{S_{n}\right\}_{n=0}^{\infty}$ is a Cauchy sequence,

$\left\|S_{n+1}-S_{n}\right\|=\left\|u_{n+1}\right\| \leq \alpha\left\|u_{n}\right\| \leq \alpha^{2}\left\|u_{n-1}\right\| \leq \cdots \leq \alpha^{n}\left\|u_{1}\right\| \leq \alpha^{n+1}\left\|u_{0}\right\|$. 
Now for $n, m \in \mathbb{N}, n \geq m$

$$
\begin{aligned}
\left\|S_{n}-S_{m}\right\| & =\left\|\left(S_{n}-S_{n-1}\right)+\left(S_{n-1}-S_{n-2}\right)+\cdots+\left(S_{m+1}-S_{m}\right)\right\| \\
& \leq\left\|S_{n}-S_{n-1}\right\|+\left\|S_{n-1}-S_{n-2}\right\|+\cdots+\left\|S_{m+1}-S_{m}\right\| \\
& \leq \alpha^{n}\left\|u_{0}\right\|+\alpha^{n-1}\left\|u_{0}\right\|+\cdots+\alpha^{m+1}\left\|u_{0}\right\| \\
& \leq\left(\alpha^{m+1}+\alpha^{m+2}+\cdots+\alpha^{n}\right)\left\|u_{0}\right\|=\alpha^{m+1} \frac{1-\alpha^{n-m}}{1-\alpha}\left\|u_{0}\right\|
\end{aligned}
$$

Hence, $\quad \lim _{n, m \rightarrow \infty}\left\|S_{n}-S_{m}\right\|=0$ that is mean $\left\{S_{n}\right\}_{n=0}^{\infty}$ is a Cauchy sequence in the Hilbert space $H$ then there exist $S \in H$ such that $\lim _{n \rightarrow \infty} S_{n}=S$, where $S=u$.

Definition 4.1 For every $n \in \mathbb{N} \cup\{0\}$, we define

$\alpha_{n}=\left\{\begin{array}{c}\frac{\left\|u_{n+1}\right\|}{\left\|u_{n}\right\|},\left\|u_{n}\right\| \neq 0 \\ 0, \text { otherwise }\end{array}\right.$

Corollary 4.1 From theorem $4.1 \sum_{i=0}^{\infty} u_{i}=\sum_{i=0}^{\infty} a_{i} \frac{(\Delta t)^{i}}{(i) !}$ convergence to exact solution $u$ when

$0 \leq \alpha_{i}<1, i=0,1,2, \ldots$

Now, to illustrate the convergence of analytical approximate solutions for the two test problems we applied Corollary 4.1 as follows;

In the first example where $(x, y) \in(-0.1,0.1)^{2}, t=1$ and $k=0.001$, for the third order solution, we get

$$
\begin{aligned}
& \alpha_{0}=\frac{\left\|u_{1}\right\|}{\left\|u_{0}\right\|}=0.06693303650<1, \\
& \alpha_{1}=\frac{\left\|u_{2}\right\|}{\left\|u_{1}\right\|}=0.03172301517<1, \\
& \alpha_{2}=\frac{\left\|u_{3}\right\|}{\left\|u_{2}\right\|}=0.02712277864<1,
\end{aligned}
$$

In the second example where $(x, y) \in(-10,10)^{2}, t=1$ and $k=0.001$ for the second order solution, we get

$$
\begin{aligned}
& \alpha_{0}=\frac{\left\|u_{1}\right\|}{\left\|u_{0}\right\|}=0.00151646710<1, \\
& \alpha_{1}=\frac{\left\|u_{2}\right\|}{\left\|u_{1}\right\|}=0.00125230375<1,
\end{aligned}
$$

hence, the convergence of approximate solutions are valid. 


\section{6- Conclusions}

In this paper, we proposed a new power series simulation scheme to solve nonlinear two-dimensional Zakharov-Kuznetsov equations. Taylor's series assisted us in the derivation of this scheme successfully. In fact, it seems that the proposed scheme can be considered as a new version of the decomposition method. The results show that a new scheme is an efficient methodology with good convergence and accuracy to find analytical approximate solutions of two test unsteady state problems. Application of the proposed approach gives a simple powerful tool to find analytic approximate solutions for the consideration problems. Finally, from analysis of results, we can conclude that the tests confirm the validity of a new scheme to handle current nonlinear problems and give the potential to employ it for more complicated problems as the future works.

\section{References}

[1] V.E. Zakharov and E.A. Kuznetsov, Three-dimensional solitons, Sov. Phys. 39(2): 285-286, 1974.

[2] A.M. Wazwaz, The extended tanh method for the Zakharov-Kuznetsov (ZK) equation, the modified ZK equation and its generalized forms, Commun. Nonlinear Sci. Numer. Simul. 13: 1039-1047, 2008.

[3] R.Y. Molliq, M.S.M. Noorani, I. Hashim and R.R. Ahmad, Approximate solutions of fractional ZakharovKuznetsov equations by VIM, J. Comput. Appl. Math. 233: 103-108, 2009.

[4] A. Yildirim and Y. Gülkanat, Analytical approach to fractional Zakharov-Kuznetsov equations by He's homotopy perturbation method, Commun. Theor. Phys. 53(6): 1005-1010, 2010.

[5] C.M. Khalique and K.R. Adem, Exact solutions of the $(2+1)$-dimensional Zakharov-Kuznetsov modified equal width equation using Lie group analysis, Mathematical and Computer Modelling, 54: 184-189, $\underline{2011 .}$.

[6] $\quad H$. Naher and F.A. Abdullah, The Improved $\left(G^{\prime} / G\right)$-Expansion method for the $(2+1)$ dimensional modified Zakharov-Kuznetsov equation. J. Appl. Math., 2012, ID 438928, (2012), 20 pages.

[7] M. Arshad, A. Seadawy, D. Lu and J. Wang, Travelling wave solutions of generalized coupled Zakharov-Kuznetsov and dispersive long wave equations, Results in Physics, 6: 1136-1145, 2016.

[8] W. Yuan, Y. Huang and Y. Shang, All traveling wave exact solutions of two nonlinear physical models. Appl. Math. Comput., 219: 6212-6223, 2013.

[9] A.R. Seadawy, Stability analysis for Zakharov-Kuznetsov equation of weakly nonlinear ion-acoustic waves in a plasma, Computers and Mathematics with Applications, 67: 172-180, 2014.

[10] S.S. Ray and S. Sahoo, New exact solutions of fractional Zakharov-Kuznetsov and modified ZakharovKuznetsov equations using fractional sub-equation method, Commun. Theor. Phys. 63(1): 25-30, 2015.

[11] J. Ahmad, G. Mohiuddin, Q.M.U. Hassan and M. Shakeel, Approximate solution of ZakharovKuznetsov equation via homotopy perturbation method, ANNALS of Faculty Engineering HunedoaraInternational Journal of Engineering, 15: 113, 2017.

[12] Z. Dong and L. Wang, Solitary wave solutions and rational solutions for modified ZakharovKuznetsov equation with initial value problem, Scientific Research Publishing, 6: 949-959, 2018.

[13] S. Monro and E.J. Parkes, The derivation of a modified Zakharov-Kuznetsov equation and the stability of its solutions, J. Plasma Phys. 62(3): 305-317, 1999. 
[14] S. Monro and E.J. Parkes, The stability of solitary-wave solutions to a modified Zakharov-Kuznetsov equation, J. Plasma Phys. 64: 411-426, 2000.

[15] A. Wiwatwanich, D. Poltem and S. Srimongkol, A novel technique for series solutions to a class of initial value problems. Global Journal of Pure and Applied Mathematics, 11(6): 4053-4061, 2015.

[16] M. Inc, Exact solution with solitary patterns for the Zakharov-Kuznetsov equations with fully nonlinear dispersion, Chaos Solitons Fractals 33: 1783-1790, 2007.

[17] A. Prakash, M. Kumar and D. Baleanu, A new iterative technique for a fractional model of nonlinear Zakharov-Kuznetsov equations via Sumudu transform, Appl. Math. Comp., 334: 30-40, 2018.

[18] T. Nawaz, A. Yıldırım and S.T. Mohyud-Din, Analytical solutions Zakharov-Kuznetsov equations, Advanced Powder Technology 24: 252-256, 2013. 